\title{
On Perron's Method for the Semi-Linear Hyperbolic System of Partial Differential Equations in Two Independent Variables
}

\author{
By Mitio Nagumo and Yukio Anasako
}

We use the notation $\partial_{x} u$ for $\frac{\partial u}{\partial x}, \partial_{x y}^{2} u$ for $\frac{\partial^{2} u}{\partial x \partial y}$, and write $u$ for $u_{1}, u_{2}$ $\cdots, u_{k}, f(x, y, u)$ for $f\left(x, y, u_{1}, u_{2}, \cdots, u_{k}\right) . \quad f(x, y)$ is said to be of $C^{1}$ class in a region $D$ if $f(x, y)$ and all its first partial derivatives are continuous in $D$. In this note we shall consider the system of partial differential equations

$$
\partial_{x} u_{i}=\lambda_{i}(x, y) \partial_{y} u_{i}+f_{i}(x, y, u)(i=1,2, \cdots, k)
$$

where variables and functions are all real valued.

O. Perron ${ }^{1)}$ had discussed the Cauchy problem for the system of equations (1) under the condition that $\lambda_{i}, f_{i}, \partial_{y} \lambda_{i}, \partial_{y} f_{i}, \partial_{u_{\mu}} f_{i}, \partial_{y u_{\mu}}^{2} f_{i}$, $\partial_{u, u \nu}^{2} f_{i}(i, \mu, \nu=1, \cdots, k)$ exist and are continuous in some region respectively.

The purpose of this note is to give such an elementary proof for the existence of solution of (1) as by Perron but under weaker assumption. We assume only the continuity of the first derivatives of $\lambda_{i}, f_{i}$ except for $\partial_{x} \lambda_{i}, \partial_{x} f_{i}$ while the proof goes merely by a modification of Perron's method.

Recently A. Douglis ${ }^{2)}$ proved the existence of the solution of equations of much more general type where is assumed only the continuity of the first derivatives of the functions in the form of equations. Our result is only a special case of Douglis' theorem, but it may be not insignificant to give an essentially simpler proof for this case.

1. As in Perron's theorem the proof of our theorem is also based on the following

1) “Über Existenz und Nichtexistenz von Integralen partieller Differentialgleichungssysteme in reellen Gebieten". Math. Zeit. 27 549-564 (1928).

2) "Some existence theorems for hyperbolic systems of partial differential equations in two independent variables". Commun. on Pure \& Appl. Math. 5 (1952), 119-154. See also: K. O. Friedrichs : "Nonlinear hyperbolic differential equations for functions of two independent variables". Amer. Jour. of Math. 70 (1948), 558-589. 
Lemma : Let $\lambda(x, y), f(x, y), \partial_{y} \lambda(x, y), \partial_{y} f(x, y)$ be continuous in

$$
B_{0}: 0 \leqq x \leqq a,|y|+K x \leqq b
$$

and suppose

$$
|\lambda(x, y)| \leqq K,\left|\partial_{y} \lambda(x, y)\right| \leqq L,|f(x, y)| \leqq g(x),\left|\partial_{y} f(x, y)\right| \leqq h(x),
$$

where $a, b, K, L$ are positive constants and $g(x), h(x)$ are integrable in $0 \leqq x \leqq a$. Let $\varphi(y)$ be of class $\boldsymbol{C}^{1}$ in $|y| \leqq b$.

Then there exists one and only one function $u(x, y)$ such that

(i) $u(x, y)$ is of class $C^{1}$ in $B_{0}$ and $u=u(x, y)$ is a solution of the equation

(ii) $u(0, y)=\varphi(y)$ for $|y| \leqq b$.

$$
\partial_{x} u=\lambda(x, y) \partial_{y} u+f(x, y) \quad \text { in } B_{0}
$$

(iii) $|u(x, y)-\Phi(x, y)| \leqq \int_{0}^{x} g(\xi) d \xi$,

$$
\left|\partial_{y} u(x, y)-\partial_{y} \Phi(x, y)\right| \leqq e^{L x} \int_{0}^{x} h(\xi) d \xi
$$

where $\Phi(x, y)$ is the solution of $\partial_{x} \Phi=\lambda(x, y) \partial_{y} \Phi$ with the initial condition $\Phi(0, y)=\varphi(y){ }^{3)}$

The proof remains essentially the same as in Perron's work ${ }^{4}$.

2. Our object is to prove

Theorem 1. Let $\phi_{i}(y)$ be of class $\boldsymbol{C}^{1}$ in $|y| \leqq b$, and $\Phi_{i}(x, y)$ be the solution of $\partial_{x} \Phi=\lambda_{i}(x, y) \partial_{y} \Phi$ such that $\Phi_{i}(0, y)=\Phi_{i}(y)$. Let $\lambda_{i}(x, y)$ and $f_{i}(x, y, u)(i=1, \cdots, k)$ be continuous in

$$
\begin{aligned}
& B_{0}: 0 \leqq x \leqq a,|y|+K x \leqq b \quad \text { and } \\
& B_{1}: \quad 0 \leqq x \leqq a,|y|+K x \leqq b,\left|u_{i}-\Phi_{i}\right| \leqq C,(i=1, \cdots, k)
\end{aligned}
$$

respectively, and

$$
\left|\lambda_{i}(x, y)\right| \leqq K,\left|f_{i}(x, y, u)\right| \leqq M
$$

where $a, b, c, K$ and $M$ are positive constants.

Let $\partial_{y} \lambda_{i}(x, y), \partial_{y} f_{i}(x, y, u), \partial_{u j} f_{i}(x, y, u)(i, j=1, \cdots, k)$ exist and be continuous in $B_{0}, B_{1}, B_{1}$ respectively.

Then there exists exactly one set of functions $u_{i}(x, y)(i=1, \cdots, k)$ such that

(i) $u_{i}(x, y)$ is of class $\boldsymbol{C}^{1}$ in

$$
B_{0}^{\prime}: 0 \leqq x \leqq l,|y|+K \leqq b \text { where } l=\operatorname{Min}\left(a, \frac{c}{M}\right) .
$$

3) $\Phi(x, y)$ is of class $C^{1}$ in $B_{0}$, the existence of $\Phi(x, y)$ is also clear.

4) ibid. (1) 
And $\left|u_{i}(x, y)-\Phi_{i}(x, y)\right| \leqq c$.

(ii) $u_{i}=u_{i}(x, y)$ is a solution of the system of differential equations

$$
\partial_{x} u_{i}=\lambda_{i}(x, y) \partial_{y} u_{i}+f_{i}(x, y, u) \quad(i=1, \cdots, k) .
$$

(iii) $u_{i}(0, y)=\varphi_{i}(y)$ for $|y| \leqq b$.

3. We prove the theorem by method of successive approximations. Set $u_{i, 0}=\Phi_{i}(x, y) \quad(i=, \cdots, k)$ and define $u_{i, n+1}(x, y)$ by the recursion's formula

$$
\begin{aligned}
& \quad \partial_{x} u_{i, n+1}=\lambda_{i}(x, y) \partial_{y} u_{i, n+1}+f_{i}\left(x, y, u_{n}\right) \\
& \text { with } \quad u_{i, n+1}(0, y)=\varphi_{i}(y) \quad(i=1, \cdots, k) .
\end{aligned}
$$

$u_{i, n+1}(x, y)$ exist for all $n$ and are of class $C^{1}\left[B_{0}^{\prime}\right]$. This is proved by Lemma using the inequality

$$
\left|u_{i, n+1}(x, y)-\Phi_{i}(x, y)\right| \leqq M x \leqq c \quad(i=, 1, \cdots, k) .
$$

There exist constants $L$ and $M^{\prime}$ such that $\left|\partial_{y} \lambda_{i}\right| \leqq L,\left|\partial_{y} \Phi_{i}\right| \leqq M^{\prime}$ in $B_{0}$, $\left|\partial_{y} f_{i}\right| \leqq M^{\prime},\left|\partial_{u j} f_{i}\right| \leqq M^{\prime}$ in $B_{1}$ for all $i, j$. We shall prove

(4) $\left|\partial_{y} u_{i, n}(x, y)-\partial_{y} \Phi_{i}(x, y)\right| \leqq\left(M^{\prime}+k M^{\prime 2}\right) e^{\mu_{0} x}$ in $B_{1}$ for all $n, i$

where

$$
\mu_{0}=\left(1+k M^{\prime}\right) e^{L^{a}}(>0) .
$$

Evidently (4) holds for $n=0$. If (4) is true for some $n$ then

$$
\begin{aligned}
\left|\partial_{y} f_{i}\left(x, y, u_{n}(x, y)\right)\right| & =\left|\partial_{y} f_{i}+\sum_{j=1}^{k} \partial_{u_{j}} f_{i} \cdot \partial_{y} \Phi_{j}+\sum_{j=1}^{k} \partial_{u_{j}} f_{i}\left(\partial_{y} u_{j, n}-\partial_{y} \Phi_{j}\right)\right| \\
& \leqq M^{\prime}+k M^{\prime 2}+k M^{\prime}\left(M^{\prime}+k M^{\prime 2}\right) e \mu_{0} x \\
& \leqq\left(M^{\prime}+k M^{\prime 2}\right)\left(1+k M^{\prime}\right) e^{\mu_{0} x} .
\end{aligned}
$$

Hence by Lemma and (5)

$$
\begin{aligned}
\left|\partial_{y} u_{i, n+1}-\partial_{y} \Phi_{i}\right| & \leqq e^{L^{a}}\left(M^{\prime}+k M^{\prime 2}\right) \frac{1+k M^{\prime}}{\mu_{0}} e^{\mu_{0} x} \\
& =\left(M^{\prime}+k M^{\prime 2}\right) e^{\mu_{0} x}
\end{aligned}
$$

then (4) holds for all $n$.

Thus there exists a constant $G$ such that

$$
\left|\partial_{y} u_{i, n}\right| \leqq G \quad \text { in } B_{0} \text { for all } n \text { and } i \text {. }
$$

4. Next we shall prove that the sequence $\left\{u_{i, n}\right\}$ converges uniformly for any $i$. There hold next equations

$$
\partial_{x}\left(u_{i, m+1}-u_{i, n+1}\right)=\lambda_{i}(x, y) \partial_{y}\left(u_{i, m+1}-u_{i, n+1}\right)+f_{i}\left(x, y, u_{m}\right)-f_{i}\left(x, y, u_{n}\right) \text {. }
$$


Then from $\left|\partial_{u} f_{i}\right| \leqq M^{\prime}$

$$
\left|f_{i}\left(x, y, u_{m}\right)-f_{i}\left(x, y, u_{n}\right)\right| \leqq k M^{\prime} e^{\mu_{0} x}|| u_{m}-u_{n} \mid \|,
$$

where

$$
\left\|\left|u_{m}-u_{n}\right|\right\| \equiv \operatorname{Max}_{\substack{1 \leqq i \leqq k \\(x, y) \in B_{0}^{\prime}}} e-\mu_{0} x\left\{u_{i, m}(x, y)-u_{i, n}(x, y)\right\} \mid
$$

and $\mu_{0}$ is defined by (5). Then we get from Lemma

$$
\left|u_{i, m+1}-u_{i, n+1}\right| \leqq \frac{k M^{\prime}}{\mu_{0}} e \mu_{0} x|| u_{m}-u_{n} \mid \|
$$

Thus

$$
\left\|u_{m+1}-u_{n+1}\right\| \mid \leqq \frac{k M^{\prime}}{\mu_{0}}\left\|u_{m}-u_{n}\right\|
$$

Set

$$
\alpha=\lim _{N \rightarrow \infty} \sup \lim _{m, n \geq N}^{\text {u. b. }}\left\|\left|\left\|u_{m}-m_{n}|\||\right.\right.\right.
$$

Then $0 \leqq \alpha \leqq 2 c<+\infty$, and from (7)

$$
\alpha \leqq \frac{k M^{\prime}}{\mu_{0}} \alpha, \text { where } 0 \leqq \frac{k M^{\prime}}{\mu_{0}}=\frac{k M^{\prime}}{\left(1+k M^{\prime}\right) e^{L^{a}}}<1 .
$$

Hence $\alpha=0$, namely

$$
\left\|u_{m}-u_{n}\right\| \rightarrow 0 \text { as } m, n \rightarrow \infty .
$$

Consequently $\quad\left\|u_{m}-u_{n}\right\|\left(\leqq e^{\mu_{0} a}\left\|u_{m}-u_{n}\right\|\right) \rightarrow 0$ as $m, n \rightarrow \infty$

where

$$
\begin{aligned}
& \left\|u_{m}-u_{n}\right\| \equiv \operatorname{Max}_{1 \leq i \leq k}\left|u_{i, m}(x, y)-u_{i, n}(x, y)\right| \text {, } \\
& (x, y) \stackrel{1 \leqq}{\epsilon} \in B_{0}^{\prime}
\end{aligned}
$$

i. e. $\left\{u_{i, n}\right\}$ converges uniformly in $B_{0}{ }^{\prime}$. We set then

$$
\lim _{n \rightarrow \infty} u_{i, n}=u_{i} \quad \text { (uniformly) } \quad(i=1, \cdots, k) .
$$

5. Now we shall prove the uniform convergence of $\left\{\partial_{y} u_{i, n}(x, y)\right\}$. Since $\partial_{y} f_{i}, \partial_{u_{j}} f_{i}$ are continuous in $B_{1}$ and $\left\{u_{n}\right\}$ converges uniformly in $B_{0}{ }^{\prime}$, for arbitrary $\varepsilon>0$ there exists $N(\varepsilon)$ such that for $m, n \geqq N(\varepsilon)$

$$
\begin{aligned}
& \left|\partial_{y} f_{i}\left(x, y, u_{m}\right)-\partial_{y} f_{i}\left(x, y, u_{n}\right)\right|<\varepsilon \\
& \left|\partial_{u_{j}} f_{i}\left(x, y, u_{m}\right)-\partial_{u_{j}} f_{i}\left(x, y, u_{n}\right)\right|<\varepsilon
\end{aligned} \quad(i, j=1, \cdots, k) \text { in } B_{0}^{\prime} .
$$

Now set

$$
H_{i, m, n}(x, y)=f_{i}\left(x, y, u_{m}(x, y)\right)-f_{i}\left(x, y, u_{n}(x, y)\right),
$$

then from (9) and (6)

$$
\begin{aligned}
& \left|\partial_{y} H_{i, m, n}\right| \leqq\left|\partial_{y} f_{i}\left(x, y, u_{m}\right)-\partial_{y} f_{i}\left(x, y, u_{n}\right)\right| \\
& +\sum_{j=1}^{k}\left\{\left|\partial_{u_{j}} f\left(x, y, u_{m}\right)-\partial_{u_{j}} f_{i}\left(x, y, u_{n}\right)\right|\left|\partial_{y} u_{j, m}\right|\right. \\
& \left.\quad+\left|\partial_{y} u_{j, m}-\partial_{y} u_{j, n}\right|\left|\partial_{u_{j}} f_{i}\left(x, y, u_{n}\right)\right|\right\}
\end{aligned}
$$




$$
\begin{aligned}
& \left|\partial_{y} H_{i, m, n}\right|<\varepsilon+\varepsilon k G+M^{\prime} e^{\mu_{0} x} \sum_{j=1}^{k}\left|e-\mu_{0} x\left(\partial_{y} u_{j, m}-\partial_{y} u_{j, n}\right)\right| \\
& \leqq \varepsilon(1+k G) e^{\mu_{0} x}+k M^{\prime}|| \partial_{y} u_{m}-\partial_{y} u_{n} \| e^{\mu_{0} x} .
\end{aligned}
$$

Hence we get from Lemma for $m, n \geqq N(\varepsilon)$ as $\partial_{y}\left(u_{i, m+1}-u_{i, n+1}\right)=\lambda_{i}(x, y)$ $\times \partial_{y}\left(u_{i, m+1}-u_{i, n+1}\right)+H_{i, m, n}$

$$
\left|\partial_{y} u_{, m+1}-\partial_{y} u_{, n+1}\right|<e^{L^{a}}\left\{\frac{\varepsilon(1+k G)}{\mu_{0}}+\frac{k M^{\prime}}{\mu_{0}}\left\|\partial_{y} u_{m}-\partial_{y} u_{n}\right\|\right\} e^{\mu_{0} x}
$$

then

$$
\text { (10) } \quad\left\|\partial_{y} u_{i, m+1}-\partial_{y} u_{i, n+1}\right\|<e^{L^{a}}\left\{\frac{\varepsilon(1+k G)}{\mu_{0}}+\frac{k M^{\prime}}{\mu_{0}}\left\|\partial_{y} u_{m}-\partial_{y} u_{n}\right\|\right\} .
$$

Now we set

$$
\beta=\lim _{v \rightarrow \infty} \sup 1 . \underset{m, n \geqq v}{\mathrm{u}} . \mathrm{b} .\left\|\partial_{y} u_{m}-\partial_{y} u_{n}\right\| \mid
$$

Then $0 \leqq \beta \leqq 2 G<\infty$, and from (10) $\beta \leqq \varepsilon(1+k G) e^{L^{a}} \mu_{0}^{-1}+k M^{\prime} e^{L^{a}} \beta \mu_{0}^{-1}$ or

$$
\left(1-\frac{k M^{\prime} e^{L^{a}}}{\mu_{0}}\right) \beta \leqq \frac{\varepsilon(1+k G) e^{L^{a}}}{\mu_{0}} .
$$

As $0 \leqq k M^{\prime} e^{L^{a}} \mu_{0}^{-1}<1$ from (5) and $\varepsilon>0$ is arbitrarily small we have

$$
\beta=0 \text {. i.d. } \quad\left\|\partial_{y} u_{m}-\partial_{y} u_{n}\right\| \rightarrow 0 \quad \text { as } m, n \rightarrow \infty .
$$

The uniform convergence of $\left\{\partial_{y} u_{i, n}\right\}$ in $B_{0}^{\prime}$ is thus proved. Hence we have from (8)

$$
\partial_{y} u_{i, n} \rightarrow \partial_{y} u_{i} \quad \text { (uniformly) } \quad(i==1, \cdots, k) .
$$

From (2), (8) and (11) we obtain

$$
\partial_{x} u_{i, n} \rightarrow \partial_{x} u_{i} \quad \text { (uniformly) }(i=1, \cdots, k),
$$

and from (2), (8), (11) and (12)

$$
\partial_{x} u_{i}=\lambda_{i}(x, y) \partial_{y} u_{i}+f_{i}(x, y, u) \quad(i=1, \cdots, k) .
$$

The existence of a desired system of solutions for (1) is thus proved. As the proof of the uniquness of the system of solutions is easy, we shall omit it.

6. From Theorem 1 immediately follows next

Theorem 2: Let $\lambda_{i j}(x, y)$ be of class $\boldsymbol{C}^{2}$ (except that $\partial_{x x}^{2} \lambda_{i j}(x, y)$ need not exist) in

$$
B_{0}: \quad 0 \leqq x \leqq a,|y| \leqq b
$$


where $a, b$ are positive constants.

Let the characteristic equation:

$$
\left|\begin{array}{ccccc}
\lambda_{11}-F & \lambda_{12} & \lambda_{13} \cdots \cdots \cdots & \lambda_{1 k} \\
\lambda_{21} & \lambda_{22}-F & \lambda_{23} & \cdots \cdots \cdots & \lambda_{2 k} \\
\cdot & \cdot & \cdot & \cdots \cdots \cdots & \cdot \\
\lambda_{k 1} & \cdot & \cdot & \cdots \cdots \cdots & \lambda_{k k}-F
\end{array}\right|=0
$$

have real distinct roots and let these roots $F_{i}(x, y)(i=1, \cdots, k)$ satisfy

$$
\left|F_{i}(x, y)\right| \leqq K \text {. }
$$

Let $\varphi_{i}(y)$ be of class $\boldsymbol{C}^{1}$ in $|y| \leqq b$. Let $f_{i}(x, y, u)$ be of class $\boldsymbol{C}^{1}$ (except that $\partial_{x} f_{i}$ need not exist) in

$$
B_{1}: \quad 0 \leqq x \leqq a, \quad|y| \leqq b, \quad\left|u_{i}-\phi_{i}\right| \leqq c, \quad(i=1, \cdots, k)
$$

where $c$ is a positive constant.

Then there exists exactly one system of functions $u(x, y)$ such that (i) $u_{i}(x, y)$ is of class $\boldsymbol{C}^{1}$ in

$$
B_{2}: \quad 0 \leqq x \leqq a^{\prime} \leqq a, \quad|y|+K x \leqq b
$$

where $a^{\prime}$ is a positive constant which is determined by $\lambda_{i, j}, \mathcal{P}_{i}$ and $f$. And

$$
\left|u_{i}(x, y)-\varphi_{i}(x, y)\right| \leqq c .
$$

(ii) $u_{i}=u_{i}(x, y)$ satisfies the system

$$
\partial_{x} u_{i}=\sum_{j=1}^{k} \lambda_{i j}(x, y) \partial_{y} u_{j}+f_{i}(x, y, u) \quad(i=1, \cdots, k) .
$$

(iii) $u_{i}(0, y)=\varphi_{i}(y)$ in $|y| \leqq b$.

Proof: See Perron's work ${ }^{5}$.

(Received September, 29, 1955)

5) ibid. (1) 